\title{
DETERMINANTES DA MOBILIDADE ACADÊMICA NO ENSINO SUPERIOR INTERNACIONAL
}

\author{
Caio Giusti Bianchi ${ }^{1}$ \\ Rosana Pêgas Godoy ${ }^{2}$ \\ Júlio César Bastos de Figueiredo ${ }^{3}$
}

\begin{abstract}
Resumo
O objetivo deste trabalho é discutir o impacto das distâncias geográfica e econômica, e diferenças de língua e renda entre o país de origem e de destino na mobilidade acadêmica internacional. Para isto, foi feita uma pesquisa quantitativa baseada em dados secundários em um modelo estrutural de regressão múltipla. As variáveis consideradas possuem impacto significativo $\left(p_{i} \leq 0,01\right)$ sobre a mobilidade acadêmica internacional com poder de explicação de 8,2\%. Por conta do aumento atual da mobilidade acadêmica internacional e seu crescente impacto no desenvolvimento econômico, os resultados compilam contribuições anteriores individuais na área e fornecem subsídios para a gestão da mobilidade acadêmica em instituições e governos.
\end{abstract}

Palavras-chave Gestão internacional. Mobilidade acadêmica internacional. Ambiente institucional.

\begin{abstract}
The aim of the research is to discuss about the impact of geographic and economic distances and language and income differences between origin and destination countries in academic mobility. A quantitative research was conducted based on secondary data in a multiple regression structural model. The variables considered have a significant impact $\left(p_{i} \leq 0,01\right)$ on international academic mobility with an $8.2 \%$ explanatory power. Due to the current rise of international academic mobility and its growing impact on economic development, the results assemble previous individual contributions in the area and provide a basis for academic mobility management in institutions and governments.
\end{abstract}

Keywords International business. International academic mobility. Institutional environment.

\section{INTRODUÇÃO}

Um dos pilares da internacionalização da educação é chamado de educação "entre fronteiras", ou transfronteiriça, caracterizado pelo movimento de estudantes entre países

\footnotetext{
${ }^{1}$ Escola Superior de Propaganda e Marketing (ESPM). Email: caio.bianchi@espm.br

${ }^{2}$ Escola Superior de Propaganda e Marketing (ESPM). Email: rrpegas@gmail.com

${ }^{3}$ Escola Superior de Propaganda e Marketing (ESPM). Email: jfigueiredo@espm.br
}

Revista de Administração Educacional, Recife, V. 1 . № 1 . 2017 jan./jun. 2017 p.04-18 
(CROWTHER et al., 2000). Deste modo, o fenômeno da mobilidade acadêmica também incorpora a proposta das novas mobilidades de Urry (2007), que ultrapassa dimensões físicas, corporais e econômicas do movimento, e alcançando dimensões culturais, afetivas, espaciais e individuais; levando a novos estudos sobre a mobilidade estudantil (ALTBACH et al., 20049).

Os valores absolutos da mobilidade acadêmica internacional aumentaram na década de 1990 por conta do avanço da globalização, justificando iniciativas científicas voltadas para compreensão do processo de mobilidade acadêmica internacional (UNESCO, 2014a; b). A assimetria entre países e sua estrutura são aspectos relevantes para a mobilidade acadêmica internacional. A assimetria pode ser observada em diferentes aspectos, como econômicos, governamentais e socioculturais, porém, de acordo com o escopo da pesquisa, as instituições formais são destacadas.

De acordo com North (1990), baseado na teoria de Ambiente Institucional, as instituições formais possuem um papel relevante na tomada de decisões em diferentes contextos em um país, sejam elas empresariais ou de mobilidade acadêmica. As instituições formais podem ser consideradas como a presença governamental, o desenvolvimento econômico e as características oficiais da região originárias do governo, como a língua oficial e a regulação governamental.

Com base em tais aspectos, o objetivo do artigo é discutir o impacto das distâncias geográfica e econômica, e diferenças de língua e renda entre o país de origem e de destino na mobilidade acadêmica internacional. Para alcançar tal objetivo, foi realizada uma pesquisa quantitativa por meio de uma regressão linear múltipla com dados secundários do fluxo de estudantes entre 133 países, considerando diferentes aspectos institucionais como variáveis independentes e a mobilidade acadêmica internacional como a variável dependente. De acordo com os resultados do modelo de regressão, quatro variáveis independentes são capazes de explicar, com $\mathrm{r}^{2}$ de 0,082, significância e confiabilidade, o fenômeno complexo e multifacetado da mobilidade acadêmica internacional.

O artigo foi dividido em: 1) Revisão da literatura sobre ambiente institucional, particularmente instituições formais, e mobilidade acadêmica internacional; 2) Desenvolvimento das hipóteses de pesquisa, relacionando os autores envolvidos na construção das hipóteses e a apresentação das mesmas; 3) Metodologia de pesquisa, com o detalhamento das etapas realizadas para alcançar as variáveis independentes e dependente; 4)

\footnotetext{
${ }^{4}$ Escola Superior de Propaganda e Marketing (ESPM). Email: caio.bianchi@espm.br
} 
Apresentação e discussão de resultados, com os coeficientes do modelo de regressão e a validação das hipóteses apresentadas; e 5) Conclusão.

\section{REFERENCIAL TEÓRICO}

\subsection{Ambiente Institucional}

A gestão internacional conta com abordagens teóricas que não se limitam ao escopo de empresas, permitindo aplicações em movimentos migratórios e mobilidade acadêmica, por exemplo. A articulação teórica entre áreas do conhecimento é representada na presente pesquisa por meio da visão baseada em instituições (intitution-based view) - ou institucionalismo -, que surgiu com o objetivo de suprir as lacunas das visões baseadas na indústria e em recursos (PENG, 2002). Há três autores principais responsáveis pela construção da área: North (1990), Scott (1995) e Peng et al. (2009). Os autores se preocuparam em criar uma taxonomia das instituições existentes em uma dada região com recortes diferentes, porém mantendo o desenvolvimento da área de conhecimento e integrando diferentes visões.

No que tange a definição de instituições no escopo do Ambiente Institucional, é possível destacar três definições principais em abordagens diferentes. Scott (1995), apoiado em uma vertente da sociologia, define instituições como estruturas com função reguladora que podem ser divididas entre normativas e cognitivas, com o objetivo de oferecer estabilidade para o comportamento em sociedade.

North (1990) e o relatório anual do Banco Mundial (1999) se apoiam em uma vertente econômica e positivista do Ambiente Institucional e ilustram o recorte a ser utilizado na presente pesquisa. Instituições então são definidas como o conjunto de restrições, oriundas do ambiente em que se encontram, de diferentes interações humanas, sejam elas formais ou informais, e responsáveis pelo desenvolvimento econômico de uma dada região.

Uma das principais características da visão institucional é a compreensão de que as tomadas de decisão de uma empresa não são oriundas apenas da economia (visão baseada na indústria) ou disponibilidade de recursos (visão baseada nos recursos), e sim pelo contexto em que a empresa se encontra, considerando as dinâmicas entre as instituições e a empresa (PENG et al., 2009). O nível de análise da visão institucional pode ser expandido para a tomada de decisão sobre a mobilidade acadêmica, especificamente na educação superior, considerando que o ambiente institucional de uma região é capaz de atrair mais ou menos estudantes

Revista de Administração Educacional, Recife, V. 1 . № 1 . 2017 jan./jun. 2017 p.04-18 
estrangeiros para o desenvolvimento acadêmico na mesma (KEHM E TEICHLER, 2007; CORBETT, 2011).

Então, as instituições são consideradas uma das responsáveis por nortear a tomada de decisão empresarial e de mobilidade acadêmica, onde é possível que o agente identifique a extensão da legitimidade das decisões, reduzindo assim a incerteza e consequentemente ampliando os resultados das mesmas.

No que tange a classificação de instituições, o recorte utilizado é apresentado por North (1990), que classifica instituições como formais ou informais. As instituições formais, de acordo com o autor, são facilmente observadas, como a situação econômica e o ambiente político da região, enquanto as instituições informais são compostas pelos níveis individual e coletivo da sociedade que integra o ambiente.

\section{Mobilidade Acadêmica Internacional}

Diante do argumento que a década de 1990 foi marcada pela abertura do mercado em diferentes regiões, paralelamente, a globalização do ensino superior foi marcada pela mobilidade de estudantes. Dados da Unesco (2014b) em 2012 ilustram que mais de 4 milhões de estudantes foram para exterior para estudar, um crescimento de 1,8\% referente ao ano de 2000, o que justifica a criação de iniciativas voltadas para compreensão dos fatores que incentivam a mobilidade de estudante entre países.

O fenômeno da mobilidade acadêmica formou uma nova onda de estudos pertinente ao assunto. A análise das influências institucionais entre os países envolvidos na mobilidade acadêmica internacional, que podem ser representados por pessoas, organizações, meio ambiente e os aspectos geográficos e econômicos dos países, passaram a ser aspectos relevantes a serem analisados na área (АLтBACH et al., 2009).

$\mathrm{Na}$ perspectiva das novas mobilidades de Urry (2007), onde a mobilidade é um fenômeno social que ultrapassa as dimensões físicas, corporais e econômicas, envolvendo também as dimensões culturais, afetivas, espaciais e individuais, as motivações de saída de um país ultrapassam as literaturas existentes sobre a mobilidade acadêmica que focam apenas em dimensões pessoais, acadêmicas e profissionais (LIMA E RIEGEL, 2012). Compreender a mobilidade por meio de aspectos institucionais formais, então, possibilita complementar a natureza do processo.

As razões pelas quais os estudantes se mobilizam para completar ou iniciar o ensino superior fora de seu país de origem ultrapassam questões do fortalecimento de capital humano

Revista de Administração Educacional, Recife, V. 1 . № 1 . 2017 jan./jun. 2017 p.04-18 
(BEINE et al., 2014). As razões também versam sobre a oferta de emprego em seu país de origem e o incentivo do governo em oferecer um ensino de qualidade em geral. Assim, estudantes que não encontram apoio para suas demandas em seu país de origem assumem a responsabilidade de busca-lo em outros países, de acordo com as Figuras 1 e 2.

Figura 1: Distribuição de mobilidade acadêmica por região geográfica de origem

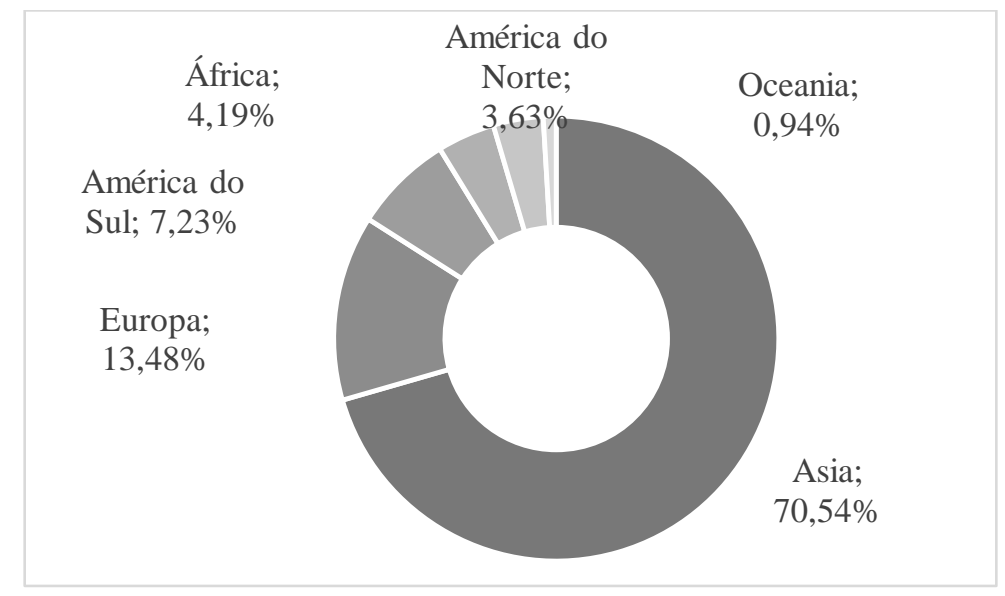

Fonte: (UNESCO, 2014b)

Figura 2 - Distribuição de mobilidade acadêmica por região geográfica de destino

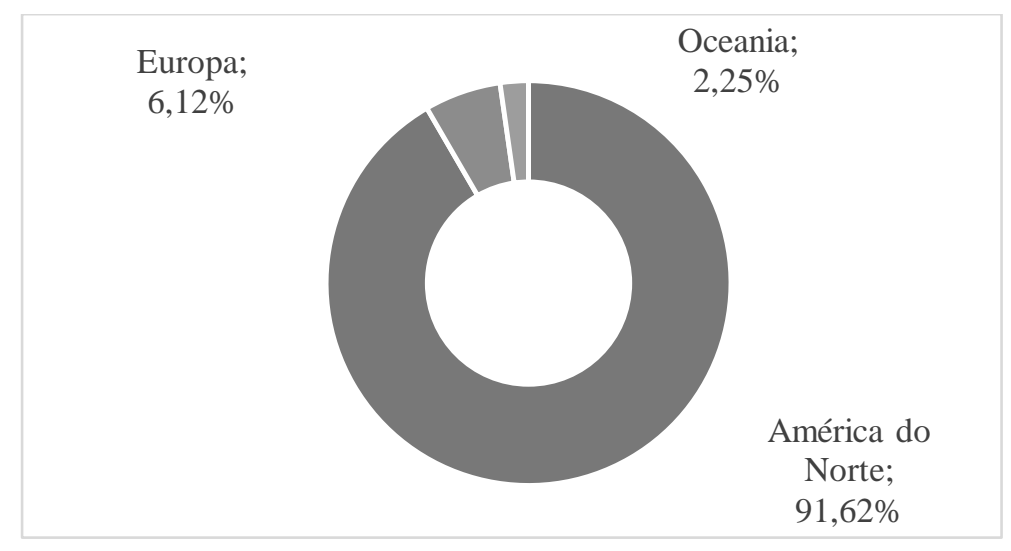

Fonte: (UNESCO, 2014b)

Poucos países desenvolvem programas que incentivam a mobilidade efetivamente, como a validação de diplomas entre universidades, disponibilidade de informações que facilitem a entrada de estudantes em outros país, e outros aspectos que facilitam a mobilidade acadêmica internacional (CONTEL E LIMA, 2008). A mobilidade é observada em grande parte na transição entre blocos economicamente concisos, como é o caso do Programa Erasmus,

Revista de Administração Educacional, Recife, V. 1 . № 1 . 2017 jan./jun. 2017 p.04-18 
que desde meados de 1980 promove a mobilidade de estudantes entre países da União Europeia (ENDERS, 1998).

Assumindo a assimetria na relação entre a entrada e saída de estudantes em busca do ensino superior (LIMA E CONTEL, 2011a), a natureza do processo de mobilidade passa por questões das novas mobilidades, que incrementa o assunto com novas tecnologias de transporte e informação (URRY, 2007). Além de haver relevância em questões econômicas, sociais, políticas e espaciais, a mobilidade acadêmica internacional não corresponde somente a bens e serviços (GIDDENS, 1990), mas é “(...) resultado de um movimento internacional de jovens ávidos para experimentar, coletivamente, formas de comunicação diferentes daquelas que as mídias clássicas nos propõem" (LEVY, 2010, p.9)

Diante do fenômeno da globalização e dos dados disseminados pelo Instituto de estatística da Organização das Nações Unidas para a Educação, Ciência e Cultura (UNESCO), os dados indicam uma assimetria entre a mobilidade de estudantes, já que os números apontam que $91,62 \%$ dos estudantes procuram a América do Norte como destino da busca pela educação formal.

Além da assimetria na mobilidade de estudantes em busca de uma educação formal, qual seria a influência das instituições formais como motivadores de escolha de um país hospedeiro? Além dos motivadores multiculturais como aprendizagem de uma nova língua e a interculturalidade (CONTEL E LIMA, 2008; GONZÁLEZ et al., 2011; BEINE et al., 2014), aspectos institucionais formais influenciam a decisão de um estudante? Deste modo, hipóteses foram formuladas.

\section{HIPÓTESES DA PESQUISA}

Estudos sobre a motivação da mobilidade acadêmica internacional (ENDERS,1998; GONZALES et al; 2011; LIMA, 2011; LIMA e RIEGEL; 2012) mostram que há motivações de ordem sociocultural, acadêmica, econômico-comercial e político administrativo. De acordo com Contel e Lima (2008), e considerando o escopo de instituições formais da pesquisa, há dois aspectos entre o país de origem e de destino que influenciam a mobilidade acadêmica: a língua oficial do país e a proximidade geográfica.

Sendo assim, a influência de questões geográficas ultrapassam questões de ordem financeira, uma vez que o nível de preços dos países de acolhimento acaba por ser determinante sobre a escolha do país de destino, principalmente sobre os custo de transporte (GONZÁLEZ et al., 2011). Tal custo de transporte, de acordo com o autor, é um dos fatores que influencia a

Revista de Administração Educacional, Recife, V. 1 . № 1 . 2017 jan./jun. 2017 p.04-18 
mobilidade acadêmica internacional, permitindo assim a formulação da primeira hipótese de pesquisa:

\section{H1: Quanto maior a distância geográfica, menor a mobilidade acadêmica.}

De acordo com Beine et al. (2014), um estudante se mobiliza em busca de fortalecer seu capital humano, mas principalmente buscando um país que possua instituições formais econômicas capazes de oferecer oportunidades nos âmbitos acadêmico e profissional. Outro aspecto que corrobora a relação é a assimetria presente em dados estatísticos, onde a saída de estudantes se concentra no hemisfério sul com destino no hemisfério norte. Com base em tais fatores, é possível levantar a segunda hipótese:

\section{H2: Quanto maior a distância econômica, maior a mobilidade acadêmica.}

Há indícios de uma continuidade do relacionamento de países pós-coloniais e sua colônia e a relação Norte-Sul (DE Sousa SANTOS E MENESES, 2014). Conforme colocado pelos autores Lima e Contel (2011b), a ligação preexistente entre as instituições formais do país de origem e destino, materializada no escopo da presente pesquisa no compartilhamento da mesma língua oficial em ambos os países, também possui relevância na mobilidade acadêmica. $\mathrm{O}$ fato de ambos os países compartilharem a mesma língua oficial, em termos práticos e segundo os autores supracitados, leva a uma mobilidade acadêmica reduzida pois a busca pelo aprendizado de novas línguas está incorporado na vivência internacional almejada pelos estudantes. Considerando a afirmação dos autores, é possível levantar a terceira hipótese:

\section{H3: Países com similaridade de língua possuem menor mobilidade acadêmica entre si.}

O interesse de países com instituições formais econômicas estabelecidas e sólidas ocorre pelo fato de que os estudantes, de acordo com Beine et al. (2014), representam uma fonte de rendimento às universidades, e países são interessados em atrair estudantes estrangeiros, convergindo seus interesses em múltiplas discussões acerca do assunto, como por exemplo, o processo de Bolonha (KEELING, 2006). Além do interesse de universidades, a renda do país de destino se mostra relevante considerando que o estudante interessado na mobilidade acadêmica busca, além de aspectos acadêmicos, o fortalecimento da sua carreira por meio de oportunidades atreladas à situação econômica do país de destino (BEINE et al., 2014). Tais aspectos levam à construção da quarta hipótese:

H4: Há maior mobilidade acadêmica em direção a países com alta renda.

Revista de Administração Educacional, Recife, V. 1 . № 1 . 2017 jan./jun. 2017 p.04-18 
Considerando as quatro hipóteses construídas na seção, é possível construir um modelo de regressão a ser validado pela pesquisa. A Figura 3 ilustra as variáveis independentes e seus respectivos efeitos na variável dependente Mobilidade Acadêmica de Ensino Superior.

Figura 3 - Modelo de Regressão

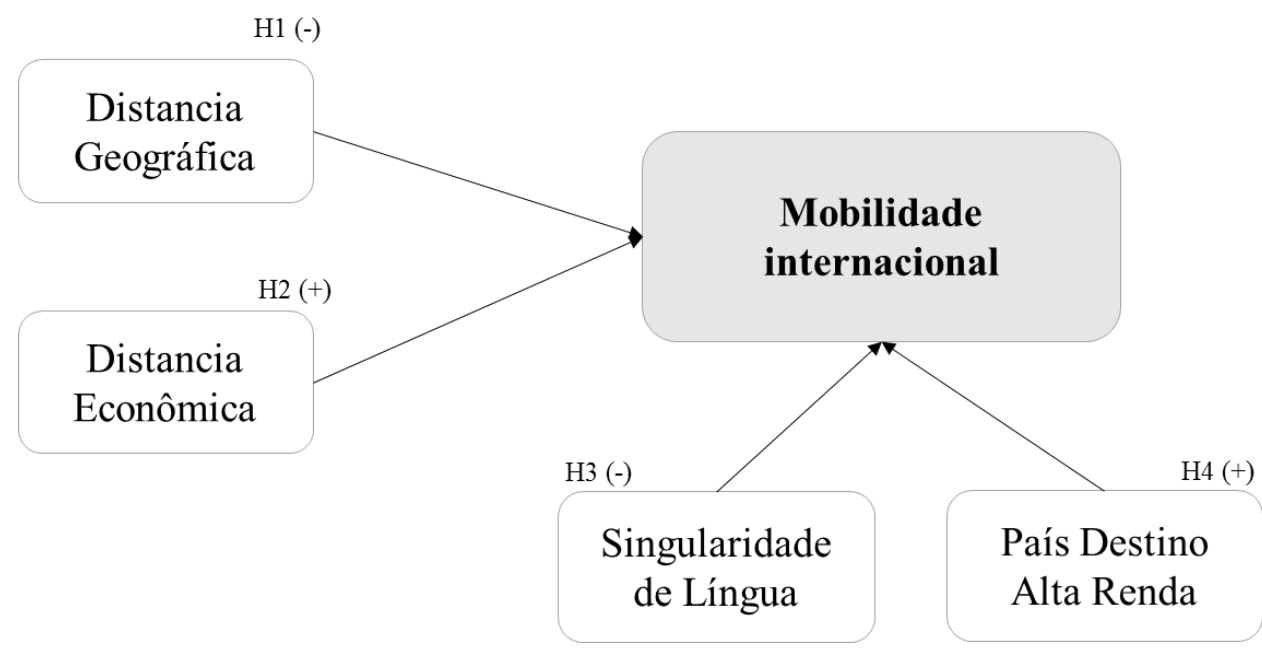

Fonte: os autores.

\section{METODOLOGIA DE PESQUISA}

As hipóteses apresentadas foram testadas utilizando uma abordagem quantitativa e a técnica de regressão linear múltipla. A regressão linear múltipla foi utilizada para verificar a existência de correlação entre a variação de dados das variáveis independentes em relação à dependente, assim como a influência exercida pelas variáveis dummy (HAIR et al, 2009).

O estudo envolve o fluxo de estudantes entre 133 países, e como um país possui fluxo de estudantes para diversos outros países, a base de dados foi formulada a partir de pares de países. O levantamento inicial de dados contemplou 13.209 pares de distâncias entre países, e como os dados são oriundos de diferentes bases de dados, os dados faltantes foram eliminados para evitar o comprometimento do modelo de regressão. Também foi realizada a padronização dos dados com base em escala logarítmica. Com ambos os procedimentos realizados, a base de dados final contou com um total de 5.779 pares de distâncias entre países.

\section{Variável Dependente}

Revista de Administração Educacional, Recife, V. 1 . № 1 . 2017 jan./jun. 2017 p.04-18 
O recorte conceitual de 'estudante internacional' utilizado na UNESCO se refere a estudantes que tenham atravessado fisicamente uma fronteira internacional com o objetivo de participar de atividades educativas no país de destino, além de a nacionalidade do estudante ser diferente de seu país de origem (UNESCO, 2014b). A base utilizada para extrair a variável dependente foi a "Inbound internationally mobile students by country of origin", do Instituto de estatística da Organização das Nações Unidas para a Educação, Ciência e Cultura (UNESCO). Os dados levantados se referem ao total de matrículas contabilizado por estudantes que entraram no país de destino.

O critério de escolha da base de dados foi definido a partir do momento em que, ao escolher a amostra de matrículas de saída, existiriam limitações quanto às especificações de registro em seu país de acolhimento. Mesmo diante do paradigma das novas mobilidades de Urry (2007), a amostra referente a mobilidade de estudantes foi feita apenas com a fatia de estudantes presenciais, desconsiderando matrículas de ensino a distância. Os dados representam a quantidade de estudantes enviados do país de origem ao país de destino, denominada mobilidade acadêmica presencial. A partir do número de matrículas de estudantes internacionais entre os países, as variáveis independentes do modelo foram construídas para posteriormente ser calculada a distância entre um ponto de dados e um centroide no espaço multivariado, ou seja, a utilização da distância mahalanobis (HAIR et al., 2009).

\section{Variáveis Independentes}

As variáveis independentes, representadas pelas distâncias calculadas a partir de dados individuais de cada país de origem e destino, são conceitualmente originadas de Berry et al. (2010).

Para o cálculo da distância geográfica entre os dois países, foram utilizadas as coordenadas geográficas do país de destino e do país de origem. O cálculo foi realizado a partir do sistema cartesiano, calculando a hipotenusa de um triângulo retângulo pelas diferenças entre latitude e longitude, pois, segundo o Teorema de Pitágoras, o quadrado da hipotenusa é igual soma dos quadrados dos catetos, remetendo aos valores sobre a distância entre os países (DOLCE E POMPEO, 2005). Os dados de geolocalização de cada país foram obtidos a partir da base de dados da CIA Factbook (2010), e não conta com divisões anuais por ser um dado característico atemporal, salvas exceções fronteiriças.

A distância econômica, segunda variável independente, foi calculada a partir da base de dados do Banco Mundial com o ano-base de 2013. O cálculo foi realizado considerando o 
Produto Interno Bruto em dólares de ambos os países, para então o cálculo da distância entre os países ser realizado. Com as três principais alternativas de distâncias analisadas (euclidiana, quadrática e mahalanobis), foi calculada a distância de Mahalanobis pelos dados da pesquisa possuírem grande variância e tal distância ser eficaz para minimiza-la por meio da matriz de variância e covariância (HAIR et al., 2009).

\section{Variáveis Dummy}

Foi possível observar na construção das hipóteses que há diferentes aspectos polarizados com impacto na mobilidade acadêmica internacional. A fim de compreender tais impactos, foram inseridas duas variáveis dummy no modelo com embasamento conceitual nas instituições formais.

A variável dummy de Similaridade de Língua, originária do trabalho de Lewer e Van Den Berg (2008), diz respeito à condição dos países de origem e de destino possuírem a mesma língua como oficial da nação. Utilizando a base de dados da CIA Factbook, com base atemporal dada a constância da língua oficial de países, o valor de 0 foi atribuído para países que possuem a mesma língua oficial e o valor de 1 para países que possuem a língua oficial diferente.

A variável dummy de País de Destino com Renda Alta representa a classificação do país de acordo com o Banco Mundial no ano de 2013, que classifica os países entre Renda Baixa, Renda Média Baixa, Renda Média Alta e Renda Alta. Com base no levantamento da literatura e na taxonomia do Banco Mundial, a dummy foi formada para que, quando o país de destino for de renda alta, possui o valor de 1, e quando for de outra classificação de renda, o valor de 0 . O Quadro 1 resume a construção das variáveis com os dados coletados e suas respectivas fontes.

Quadro 1 - Construção das Variáveis

\begin{tabular}{cccc}
\hline VARIÁVEL & AUTOR & MEDIDA & FONTE \\
\hline Mobilidade & Beine, Noël e & Quantidade de estudantes & UNESCO \\
Acadêmica (D) & Ragot (2014) & (2013) \\
\hline $\begin{array}{c}\text { Distância Geográfica } \\
\text { (I) }\end{array}$ & Lewer (2008) & Quilômetros entre países & CIA Factbook \\
Distância Econômica & Berry, Guillén e & (atemporal) \\
(I) & Zhou (2010) & (Produto Interno Bruto) & Banco Mundial \\
\hline
\end{tabular}

Revista de Administração Educacional, Recife, V. 1 . № 1 . 2017 jan./jun. 2017 p.04-18 


\begin{tabular}{cccc}
\hline $\begin{array}{c}\text { Dummy } \\
\text { Similaridade } \\
\text { de Língua }\end{array}$ & Lewer (2008) & Comunalidade da língua & CIA Factbook \\
oummy & & oficial entre países & (atemporal) \\
País Destino & Lewer (2008) & Classificação de renda do país segundo o & Banco Mundial \\
Renda Alta & & Banco Mundial & $(2013)$ \\
\hline
\end{tabular}

Fonte: Elaborada pelos autores

\section{DISCUSSÃO DOS RESULTADOS}

Antes da apresentação dos resultados da regressão, foi realizada uma análise de correlação entre as variáveis do modelo a fim de validar a consistência de dados. A Tabela 1 mostra que não há multicolinearidade no modelo proposto, considerando o critério de, no máximo 0.7 de correlação na variação dos dados com significância (HAIR et al, 2009).

Com a ausência de correlação entre variáveis verificada, a regressão linear múltipla foi realizada com suporte do software SPSS (FIELD, 2009). Os resultados da regressão podem ser observados na Tabela 2. O modelo é apropriado pois, além do teste de colinearidade, por meio dos valores de Durbin Watson, VIF e F, todos os parâmetros estão dentro do padrão e/ou com significância. A apresentação e discussão dos resultados do modelo, que conta com duas variáveis independentes e duas variáveis dummy, apresenta o $\mathrm{R}^{2}$ da ordem de 8,20\%.

O coeficiente de distância geográfica, significante com o coeficiente de $-0,423$, confirma a primeira hipótese de pesquisa de que quanto menor a distância geográfica entre dois países, maior será a mobilidade acadêmica internacional. Tal comportamento pode ser justificado conceitualmente pelo valor pago ao movimento espacial que o estudante necessita realizar, tanto quanto os custos envolvidos com tal processo no país de acolhimento.

Revista de Administração Educacional, Recife, V. 1 . № 1 . 2017 jan./jun. 2017 p.04-18 
Tabela 1 - Matriz de Correlações

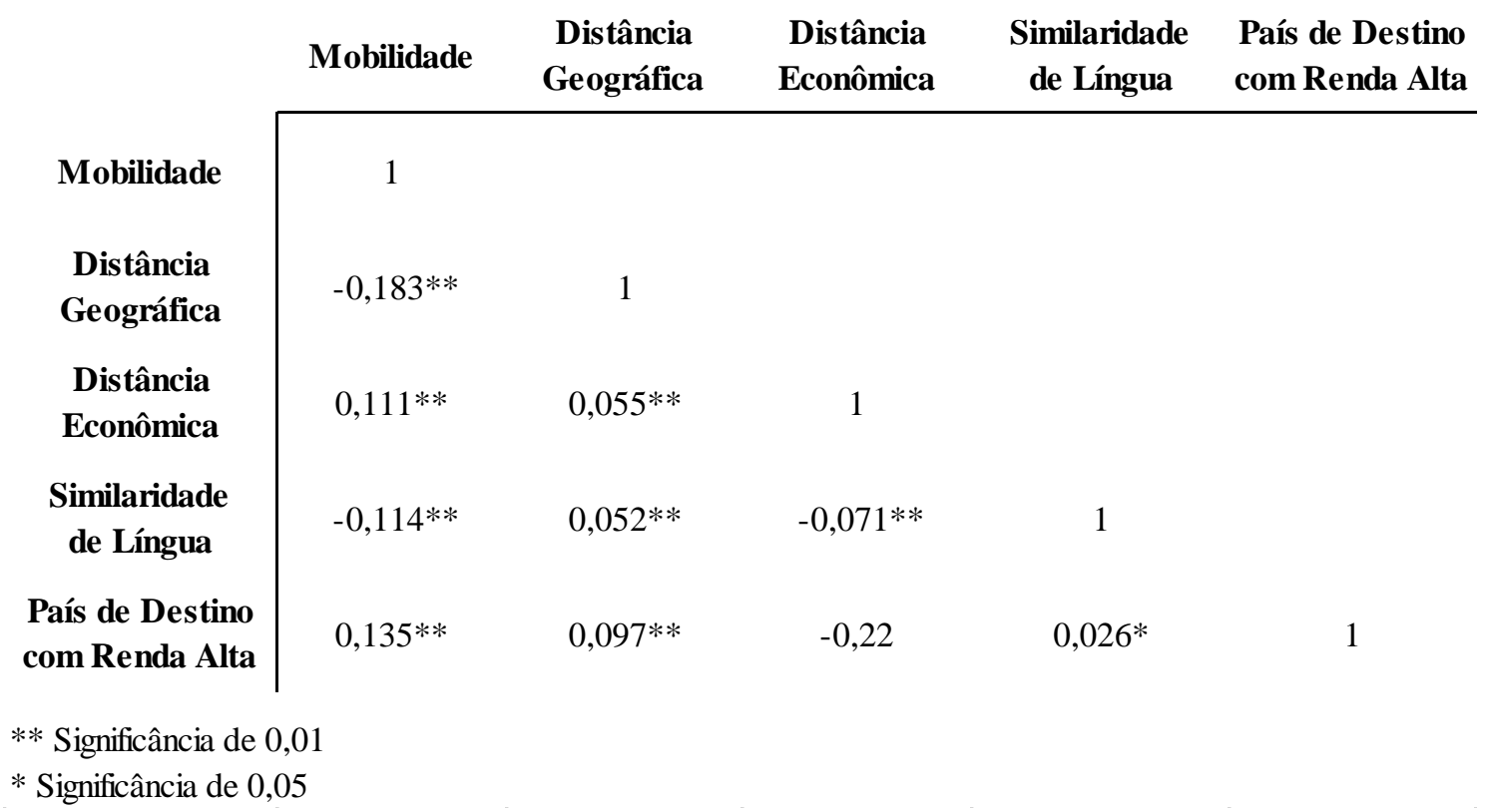

O coeficiente de distância econômica, significante com coeficiente de 0,217 , confirma a segunda hipótese de pesquisa, onde a assimetria econômica entre países faz com que haja maior mobilidade acadêmica. Conforme observado na revisão teórica, o volume intercambiado entre países não corresponde tão somente a bens e serviços, mas pelas novas mobilidade desenvolvidas por novas tecnologias de transporte e informação. A globalização afeta a expansão do ensino superior e consequentemente a mobilidade acadêmica de países em desenvolvimento para países desenvolvidos, buscando educação de maior qualidade.

A dummy de língua, significante com coeficiente negativo de 0,422 confirma a terceira hipótese de pesquisa. De acordo com a literatura e a concepção das hipóteses, o fato de o país de origem e de destino possuírem a mesma língua falada oficialmente faz com que haja uma redução na mobilidade acadêmica. O motivo de tal redução se dá pelo fortalecimento da concepção de que o movimento de estudantes é marcado pela vontade de novos experimentos socioculturais, levando em conta a diversidade da linguística e diversidade sócio cultural.

A dummy de renda, que conta com o valor de 1 quando há movimento de estudantes para países de renda alta, possui coeficiente de 0,367 , o que confirma a quarta hipótese de pesquisa. Tal hipótese considera que o fato de o país de destino ser de alta renda faz com que haja maior mobilidade acadêmica em direção ao mesmo. A diferença de renda entre os países 
pode ser interpretada por meio das possibilidades de mercado ofertadas pelo país de destino, e também pela estrutura institucional formal que é englobada pelo mesmo.

Tabela 2 - Coeficientes de Regressão

\begin{tabular}{ccc} 
Variável & Coeficiente & VIF \\
\hline DISTÂNCIA GEOGRÁFICA & $-0,423 * *$ & 1,016 \\
DISTÂNCIA ECONÔMICA & $0,217 * *$ & 1,009 \\
SIMILARIDADE LÍNGUA & $-0,422 * *$ & 1,009 \\
PAÍS DESTINO RENDA ALTA & $0,367 * *$ & 1,011 \\
\hline $\mathrm{R}^{2}$ & $8,20 \%$ & \\
$\mathrm{~F}$ & $130,015 * *$ & \\
Durbin-Watson & 1,879 & \\
$* *$ Significância de 0,01 & & \\
$*$ Significância de 0,05 & &
\end{tabular}

Definindo a $M O B I L I D A D E_{i j}$ entre países $i$ e $j, D I S T_{G E O}$ como a distância entre os países $i$ e $j$, $D I S T_{G P D}$ como a distância entre o produto interno bruto dos países $i$ e $j$, e finalmente as variáveis dummy, DUMMY $Y_{\text {LINGUA }}$ com a similaridade de línguas e DUMMY $Y_{D E S T}$ RENDA ALTA quando o destino do estudante é um país de renda alta, o modelo pode ser resumido na equação abaixo:

$$
\begin{aligned}
& M O B I L I D A D E_{i j}=2,995-D I S T_{G E O} * 0,423+D I S T_{G P D} * 0,217-D U M M Y_{L I N G U A} * \\
& 0,422+D U M M Y_{\text {DEST RENDA ALTA }} * 0,367
\end{aligned}
$$

\section{CONCLUSÃO}

De acordo com o objetivo da pesquisa de discutir o impacto das distâncias geográfica e econômica, e diferenças de língua e renda entre o país de origem e de destino na mobilidade acadêmica internacional; aliado aos resultados observados no modelo de regressão apresentado, é possível afirmar que há uma relação entre as distâncias geográfica e de instituições formais com a mobilidade acadêmica internacional. A influência de tais instituições reflete características específicas da mobilidade, como a busca por regiões com

Revista de Administração Educacional, Recife, V. 1 . № 1 . 2017 jan./jun. 2017 p.04-18 
instituições formais sólidas capazes de oferecer uma experiência acadêmica e profissional relevante para os estudantes.

Os dados empíricos coletados e analisados no artigo, além de confirmarem as hipóteses levantadas, são consonantes com as afirmações embasadas na literatura. A mobilidade acadêmica possui movimentos internacionais específicos que são regidos, de maneira geral e paradoxal, pelo interesse em aspectos semelhantes ao país de origem - como a distância geográfica - e também pela motivação em aspectos opostos ao país de origem como a língua e aspectos econômicos.

Os dados mostram que o fenômeno da mobilidade acadêmica internacional não é limitado aos estudos dos indivíduos, e sim que há um transbordamento do interesse para além da academia. A mobilidade, como pode ser observada no maior fluxo em países com rendas e economicamente diferentes, abrange o interesse dos indivíduos em tornar a experiência financeiramente sustentável.

A literatura voltada para as motivações da mobilidade acadêmica internacional, por vezes, é esparsa. Com base na dispersão de achados de diferentes autores, a contribuição acadêmica do artigo se faz relevante ao consolidar tais motivações oriundas da literatura em um mesmo estudo. Além de consolidar diferentes autores, o artigo possui a contribuição de provar, quantitativamente, que há relação entre as distâncias geográficas e institucionais e a mobilidade acadêmica internacional.

Como a mobilidade acadêmica internacional é um aspecto de interesse governamental para que haja diversificação na construção do conhecimento e fortalecimento econômico de um país, a contribuição de políticas públicas do artigo ilustra quatro fatores motivacionais para tal mobilidade. Com a confirmação quantitativa das motivações para o fluxo internacional de estudantes, é possível a análise de investimentos pontuais para potencializar a recepção de tais estudantes em um dado país.

O poder explicativo do modelo de regressão, de 8,2\% reforça o argumento da literatura, adotado no presente estudo, de que o fenômeno da mobilidade acadêmica internacional é multifacetado e complexo. O processo decisório de um estudante em confiar sua carreira acadêmica a uma instituição de outro país envolve diversos aspectos psicológicos, culturais, financeiros e acadêmicos, sendo as variáveis abordadas no estudo um dos aspectos do fenômeno. A limitação e oportunidade de pesquisas futuras do artigo se encontram na possibilidade de ampliação da análise para abranger diferentes variáveis independentes, como as instituições informais - normativas e cognitivas.

Revista de Administração Educacional, Recife, V. 1 . № 1 . 2017 jan./jun. 2017 p.04-18 


\section{REFERÊNCIAS}

ALTBACH, P. G.; REISBERG, L.; RUMBLEY, L. E. Trends in global higher education: Tracking an academic revolution: UNESCO Pub.; Sense 2009.

BEINE, M.; NOËL, R.; RAGOT, L. Determinants of the international mobility of students. Economics of Education review, v. 41, p. 40-54, 2014. ISSN 0272-7757.

BERRY, H.; GUILLÉN, M. F.; ZHOU, N. An institutional approach to cross-national distance. Journal of International Business Studies, v. 41, n. 9, p. 1460-1480, 2010. ISSN 0047-2506.

CONTEL, F. B.; LIMA, M. C. Aspectos da internacionalização do ensino superior: origem e destino dos estudantes estrangeiros no mundo atual. 2008. ISSN 1980-4865.

CORBETT, A. Ping Pong: competing leadership for reform in EU higher education 19982006. European Journal of education, v. 46, n. 1, p. 36-53, 2011. ISSN 1465-3435.

DE SOUSA SANTOS, B.; MENESES, M. P. Epistemologias do sul. Cortez Editora, 2014. ISBN 8524921072.

DOLCE, O.; POMPEO, J. N. Fundamentos de matemática elementar, 9: geometria plana. Atual, 2005. ISBN 853570552X.

ENDERS, J. Academic Staff Mobility in the European Community: The ERASMUS Experience. Comparative Education Review, v. 42, n. 1, p. 46-60, 1998. ISSN 00104086, 1545701X. Disponível em: 〈 http://www.jstor.org/stable/1188786 >.

FACTBOOK, C. The world factbook. See also: https://www. cia. gov/library/publications/the-world-factbook, 2010.

FIELD, A. Descobrindo a estatística utilizando o SPSS. Porto Alegre: Artmed, 2009.

GIDDENS, A. As conseqüências da modernidade. unesp, 1990. ISBN 8571390223.

GONZÁLEZ, C. R.; MESANZA, R. B.; MARIEL, P. The determinants of international student mobility flows: an empirical study on the Erasmus programme. Higher Education, v. 62, n. 4, p. 413-430, 2011. ISSN 0018-1560.

HAIR, J. F. et al. Análise multivariada de dados. Bookman, 2009. ISBN 8577805344.

KEELING, R. The Bologna Process and the Lisbon Research Agenda: the European Commission's expanding role in higher education discourse. European Journal of Education, v. 41, n. 2, p. 203-223, 2006. ISSN 1465-3435.

KEHM, B. M.; TEICHLER, U. Research on internationalisation in higher education. Journal of Studies in International Education, v. 11, n. 3-4, p. 260-273, 2007. ISSN 1028-3153.

LEVY, P. Cibercultura. Editora 34, 2010. ISBN 8573261269.

Revista de Administração Educacional, Recife, V. 1 . № 1 . 2017 jan./jun. 2017 p.04-18 
LEWER, J. J.; VAN DEN BERG, H. A gravity model of immigration. Economics letters, v. 99, n. 1, p. 164-167, 2008. ISSN 0165-1765.

LIMA, M. C.; CONTEL, F. B. Internacionalização da Educação Superior: Nações ativas, nações passivas e a geopolítica do conhecimento. 2011a. ISBN 8579390672.

Internacionalização da Educação Superior: Nações ativas, nações passivas ea geopolítica do conhecimento. 2011b. ISBN 8579390672.

LIMA, M. C.; RIEGEL, V. A Mobilidade Acadêmica no Contexto Sul-Americano: Estudantes Brasileiros e Colombianos, suas Experiências de Mobilidade Acadêmica Internacional e o Mercado de Trabalho. ANPAD - Associação Nacional de Pós Graduação e Pesquisa em Administração, v. XXXVI, 2012. Disponível em: < http://www.anpad.org.br/admin/pdf/2012_EPQ465.pdf $>$.

MUNDIAL, B. The World Bank annual report 1999. 1999

. World Economic Outlook Database. 2013. Disponível em: < http://data.worldbank.org/ >. Acesso em: 1 de Dezembro de 2015.

NORTH, D. C. Institutions, institutional change and economic performance. Cambridge university press, 1990. ISBN 0521397340.

PENG, M. W. Towards an institution-based view of business strategy. Asia Pacific Journal of Management, v. 19, n. 2-3, p. 251-267, 2002. ISSN 0217-4561.

PENG, M. W. et al. The Institution-Based View as a Third Leg for a Strategy Tripod. The Academy of Management Perspectives, v. 23, n. 3, p. 63-81, 2009. ISSN 1558-9080.

SCOTT, W. R. Institutions and organizations. Sage Thousand Oaks, CA, 1995.

UNESCO. Educação para todos 2000-2015: progressos e desafios. 2014a. Disponível em: < http://unesdoc.unesco.org/images/0023/002325/232565por.pdf $>$. Acesso em: 02 de Dezembro de 2015.

- Global Flow of Tertiary-Level Students. 2014b. Disponível em: < http://www.uis.unesco.org/Education/Pages/international-student-flow-viz.aspx $>$. Acesso em: 02 de Dezembro de 2015.

URRY, J. Mobilities. London: Polity, 2007. 335 ISBN 0745634192.

Revista de Administração Educacional, Recife, V. 1 . № 1 . 2017 jan./jun. 2017 p.04-18 